\title{
A Novel Construction and Block Error Rate Analysis of Polar Codes with Short Messgage Transmission over Different Channels
}

\author{
A Sai Naga $\mathrm{Sri}^{1} \mid$ Dr. S V R K Rao ${ }^{1}$
}

${ }^{1}$ M.Tech(DECS), Department of ECE, Godavari institute of Engineering \& Technology (A), NH-16, Chaitanya Knowledge City, Rajahmundry, Andhra Pradesh, INDIA.

Professor, Department of ECE, Godavari institute of Engineering \& Technology (A), NH-16, Chaitanya Knowledge City, Rajahmundry, Andhra Pradesh, INDIA.

\section{To Cite this Article}

A Sai Naga Sri and Dr. S V R K Rao, "A Novel Construction and Block Error Rate Analysis of Polar Codes with Short Messgage Transmission over Different Channels", International Journal for Modern Trends in Science and Technology, 6(10): 01-06, 2020.

\section{Article Info}

Received on 06-September-2020, Revised on 18-September-2020, Accepted on 25-September-2020, Published on 29-September-2020.

\section{ABSTRACT}

Polar codes are usually constructed to minimize the upper bound of a block error ratio (BLER). In this paper, we discuss the estimation of the exact BLERs of polar codes as well as the construction of polar codes. Assuming that successive cancellation (SC) decoding is employed, we present a method for estimating the exact BLER of polar codes with the help of Gaussian approximation (GA). A new approach is proposed to construct polar codes over different channels (Awgn and Rayleigh+) with short message transmission scheme, aimed at minimizing the exact BLER, instead of the upper bound of the BLER. Analysis indicates that the new approach less complex than the existing methods. It is also shown that the proposed scheme is outperforms than conventional methods and are visualized in simulation results.

Keywords: BLER, SC, GA, AWGN, Rayleigh

\section{INTRODUCTION}

With the increased future smart transportation systems, rapid mobility takes place with variable Doppler shifts with time based phenomena in wireless communication. Peak data rate problems are encountered. So we need efficient encoding and decoding schemes to increase the peak data rate in high velocity environment.Hence, $5 \mathrm{G}$ is the better candidate to attain data rates up to $20 \mathrm{~Gb} / \mathrm{s}$ for eM-BB. So, efficient coding scheme is a challengingtask in wireless $5 \mathrm{G}$ for high mobility scenario, $5 \mathrm{G}$ places attention on better-quality error-correction tedious [1].
$5 \mathrm{G}$ stands for 5 th generation. To be more

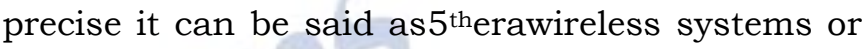
$5^{\text {th }}$ era mobile-networks. It is the planned upcoming generation standard beyond the current LTE radio access technology standards. The peak speed that would be offered by $5 \mathrm{G}$ are5Gbps for downloads. Most of the RAN planning for the $5 \mathrm{G}$ network are currently ongoing and $5 \mathrm{G}$ basic aim is to provide better capacity than current LTE, VOLTE, which allows a sophisticated mobile density for high speed network users and reliable in D2D,ultrareliable, and massive-machine connectivity[1][2]. 
Gigantic MIMO alludes to the utilization of many administration recieving wires working radio wires working reasonably and adaptively, to altogether enlarge the data transfer capacity in versatile systems, rather than the ordinary point-to-point MIMO. Monstrous MIMO assumes a significant job in upgrading the $5 \mathrm{G}$ innovation. As MIMO contains huge number of radio wires it centers the gathering and transmission of the vitality of the sign into exceptionally littler districts of room. This achieves tremendous improvement in the throughput and vitality productivity. MIMO is said to total separation with the continuous practice by utilizing many administration radio wires that are worked adaptively and rationally especially when joined. This is said to occur with synchronous booking of numerous client terminals (e.g., tens or hundreds). Additional recieving wires help by centering the transmission and gathering of sign vitality into ever-littler districts of room. This acquires enormous enhancements throughput and vitality effectiveness, in especially when joined with synchronous booking of countless client terminals. (e.g., tens or hundreds)

Polar code is a linear block error correcting code. Construction of polar code is based on the recursion of a short kernel code. This code is transforms physical channel into virtual channel .If the no of recursions are high, the reliability of virtual channels tends to either high or low .Most reliable channels are receive the data bits .Polar codes are error correcting codes which are first family of error correcting code. The errors obtain the capacity of binary input memory less and systematic channels with decoding, encoding and construction algorithms.

Polar codes being used in 5G wireless standard due to provide great error correcting performance with low decoding complexity. When combined with more advanced decoding algorithms. This makes outstanding performance.

Polar Codes are said to achieve channel capacity in a given binary discrete memory less channel. The polar code structure is to select the best $\mathrm{K}$ bit channels among $\mathrm{N}$ number of bit channels to transfer the information. By using successive cancellation list (SCL) decoding is used to improve the finite-length performance of polar codesis proposed. Encoding systematically is an alternative of the unique non-systematic encoding is another way to improve error performance of polar codes.

\section{LITERATURE SURVEY}

R.Mori,et al [2]: The creators of this paper has given a short clarification about the exhibition of polar codes. These are the principal group of codes-known to accomplish limit of symmetricchannels utilizing a low intricacy progressive crossing out decoder. Despite the fact that these codes, joined with progressive wiping out, are ideal in this regard, their limited length execution isn't record breaking.

I.Tal,et al [3]: The creators of this paper has given a short clarification about the strategy for proficiently developing polar codes is displayed and broke down. Albeit polar codes are unequivocally characterized, straight forward development is immovable since the subsequent polar piece channels have a yield letter set that develops exponentially with the code length. In this manner, the center issue that should be explained is that of dependably approximating a bit-channel with an obstinately enormous letters in order by another channel having a reasonable letter set size. They devise two estimate techniques which "sandwich" the first piece channel between a corrupted and an overhauled form thereof. The two approximations can be proficiently figured and end up being amazingly close practically speaking.

P.Trifonov [4]: The creator of this paper has given a short clarification about the proficient plan and translating of polar codes of both summed up linked codes and staggered codes. It is demonstrated that the exhibition of a polar code can be improved by speaking to it as a staggered code and applying the multistage disentangling calculation with most extreme probability deciphering of external codes. Extra execution improvement is acquired by supplanting polar external codes with different ones with better blunder adjustment execution. Sometimes this likewise results in multifaceted nature decrease. It is appeared Gaussian guess for thickness development empowers one to precisely foresee the exhibition of polar codes and connected codes dependent on them.

M.Morelli,et al [5]: The creators of this paper has given a short clarification about the pilot-supported channel estimation technique for OFDM frameworks is tended to for PACE equidistantly dispersed pilot images permit to reproduce the channel reaction by methods for insertion. The ideal least mean squared blunder (MMSE) estimator performs smoothing and 
introduction together. To lessen the intricacy of the ideal MMSE estimator, and propose to isolate the smoothing and insertion undertakings. The isolated smoothing and introduction estimator (SINE) comprises of a MMSE based smoother which just works at the got pilot images, and an interpolator which is free of the channel measurements. The isolated methodology draws near to the ideal MMSE, while the intricacy is horribly diminished.

PeiyaoChen,et al [6]: The creators of this paper has given a concise clarification about the plan and execution of polar codes for $5 \mathrm{G}$ correspondence under high versatility situations With the advancement of fast trains (HST), productive and dependable correspondence benefits in high portability situations have turned into a critical interest.

Kai Chen,et al[7]: The SC translating calculation of polar codes and its improved variants, in particular, SCL and SCS, are repeated as way search methods on the code tree of polar codes. By joining the standards of SCL and SCS, a conventional ISC deciphering plan called the $\mathrm{SCH}$ unraveling is proposed. This proposed plan can give an adaptable setup when the reality complexities are restricted. To maintain a strategic distance from superfluous way looking, a pruning method reasonable for every one of the three ISC interpreting plans is proposed. Execution and multifaceted nature examination dependent on reproductions demonstrates that in the moderate and high SNR routine, the pruned ISC decoders can approach the exhibition of ML deciphering with the reality complexities extremely near those of SC. Nadine Hussami,et al [8]: Polar codes, presented as of late by Arrkan, are the principal group of codes known to accomplish limit of symmetric channels utilizing a low multifaceted nature progressive undoing decoder. In spite of the fact that these codes, com bined with progressive dropping, are ideal in this regard, their limited length execution isn't record breaking. We talk about a few strategies through which their limited length execution can be im demonstrated. We additionally contemplate the presentation of these codes with regards to source coding, both misfortune less and lossy, in the single-client setting just as for appropriated applications.

Tao Wang,et al [9]: The creators have proposed PCC polar codes to sum up the CRC-linked polar codes. With the proposed heuristic development, the PCC polar codes show a capability of blunder execution upgrades over CRC-connected polar codes, particularly when the code word length is short and code rate is low.

\section{ORGANIZATION OF THE MANUSCRIPT}

In this proposal section 1 contains introduction, section 2 contains literature survey, section4 contains proposed model with correspond mathematical analysis, sections 5 contains results and discussions.

\section{PROPOSED MODEL OF SMT (SHORT MESSAGE TRANSMISSION)-POLAR CODES}

\section{A. POLAR CODE BASIC CHANNEL}

Before proceeding with the polar code we construct and specify a channel $\mathrm{W}_{2}$ which is a Binary discrete memoryless channel(BDMC) and it is to achieve symmetric capacity $\mathrm{I}(\mathrm{W})$ which is the highest rate and this is subjected to using the I/P letters of the channel which are equally probable[1].

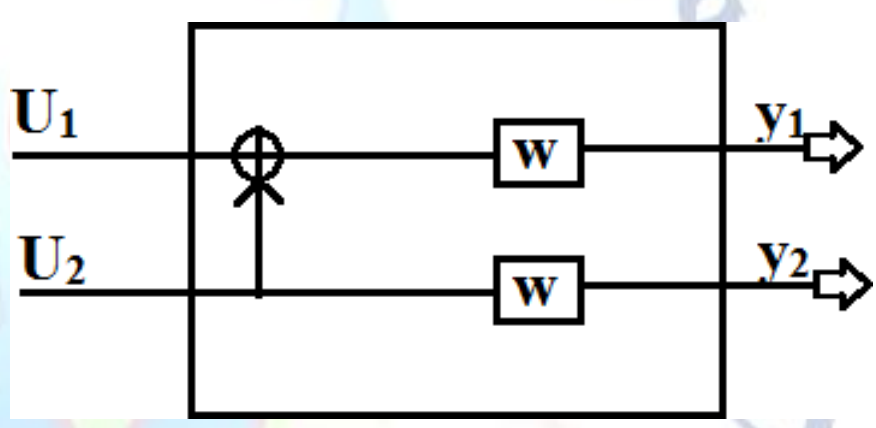

Fig1: polar code basic channelW

figure 1 shows the encoder of polar code .It is possible to synthesize or create a second set of $\mathrm{N}$ binary input channels out of Nindependent copies of a given $\mathrm{B}-\mathrm{DMCW}$ and the channels have the properties $\left\{\mathrm{W}_{\mathrm{N}}^{(\mathrm{i})}: 1<=i<=N\right\}$. One thing to be noted here is that when $\mathrm{N}$ becomes bigger, 2 things tend to happen i.e. some of the fraction of channels for the indices $I$ for $I\left(\mathrm{~W}^{(\mathrm{i})} \mathrm{N}\right)$ is near 0 approaches $1-\mathrm{I}(\mathrm{w})$ and rest of the fraction of channels for indices I for $\mathrm{I}\left(\mathrm{W}^{(\mathrm{i})} \mathrm{N}\right)$ is near 1 approaches $\mathrm{I}(\mathrm{w})$. The complexity of the decoders and encoders that achieve this performance are having a complexity of $\mathrm{O}(\mathrm{N} \operatorname{logN})$.

For a Binary Discrete memorylesschannel $\mathrm{W}$, there are basically two channel parameters of interest. One is the symmetric capacity $\mathrm{I}(\mathrm{W})$ and the second one is the Bhattacharya parameter.

$I(W)=\sum_{y \in Y} \sum_{x \in X^{2}} \frac{1}{2}(y \mid x) \log \frac{w(y \mid x)}{\frac{1}{2} w(y \mid 0)+\frac{1}{2} w(y \mid 1)}$

and the Bhattacharyya parameter is given by

$\mathrm{Z}(\mathrm{W})=\sum_{\mathrm{y} \in \mathrm{Y}} \sqrt{\mathrm{w}(\mathrm{y} \mid 0) \mathrm{w}(\mathrm{y} \mid 1)}$

(2) 
The symmetric capacity parameter is used as a measure of rate and the Bhattacharya parameter is used as a parameter of reliability, respectively.

\section{B.BLER ANALYSIS AND CONSTRUCTIONOF POLAR CODES}

\section{a. BLER ANALYSIS OF POLAR CODES WITH SC DECODING}

The block error rate of the upper bound can be denoted as

$$
P(\varepsilon)=\sum_{i \in I} P\left(B_{i}\right) \leq \sum_{i \in I} P\left(A_{i}\right) \leq \frac{1}{2} \sum_{i \in I} Z_{N}^{(i)}
$$

It is obvious that $\mathrm{B}_{i}$ is a joint event that $\hat{u}_{i} \neq u_{i}$ occurstogether with $\hat{u}_{1}^{i-1}=u_{1}^{i-1}$. However, the two events are notindependent, which makes the estimation of $P\left(B_{i}\right)$ becomehard. Here, we denote $C_{i}$ as

$C_{i} \square\left\{\hat{u}_{i} \neq u_{i} \mid \hat{u}_{1}^{i-1}=u_{1}^{i-1}\right\}$

which is the event that $\hat{u}_{i} \neq u_{i}$ under the condition that $\hat{u}_{1}^{i-1}=u_{1}^{i-1}$. Let $C_{i}^{c}$ denote the complement event of $C_{i}$. Apparently, $C_{i}^{c}$ is the event that $\hat{u}_{i}=u_{i}$ under the condition that $\hat{u}_{1}^{i-1}=u_{1}^{i-1}$ Hence, we have

$$
\begin{aligned}
& P\left(C_{i}\right)=P\left(\hat{u}_{i} \neq u_{i} \mid \hat{u}_{1}^{i-1}=u_{1}^{i-1}\right) \\
& =\frac{P\left(\hat{u}_{i} \neq u_{i} \mid \hat{u}_{1}^{i-1}=u_{1}^{i-1}\right)}{P\left(\hat{u}_{1}^{i-1}=u_{1}^{i-1}\right)} \geq P\left(B_{i}\right), \\
& P\left(C_{i}^{c}\right)=P\left(\hat{u}_{i}=u_{i} \mid \hat{u}_{1}^{i-1}=u_{1}^{i-1}\right)
\end{aligned}
$$

Let denote the event that the block is decoded correctly, which is $\hat{u}_{1}^{N}=u_{1}^{N}$. It is easy to obtain that

$$
\begin{aligned}
P\left(\varepsilon^{c}\right) & =P\left(\hat{u}_{1}^{N}=u_{1}^{N}\right) \\
& =\mathrm{P}\left(\hat{u}_{1}=u_{1}\right) \ldots . . \mathrm{P}\left(\hat{u}_{N}=u_{N} \mid \hat{u}_{1}^{N-1}=u_{1}^{N-1}\right) \\
& =P\left(C_{1}^{c}\right) P\left(C_{2}^{c}\right) \ldots \ldots . P\left(C_{N}{ }^{c}\right)
\end{aligned}
$$

Based on this, the probability that the information $u_{1}^{N}$ cannotbe decoded correctly can be computed by $P(\varepsilon)=1-P\left(\varepsilon^{c}\right)=1-\Pi\left(1-P C\left(C_{i}\right)\right)$

The block error probability given in (3) is the exact representationof the BLER of a polar code with SC decoding. In thenext subsection, we will show that
$P\left(C_{i}\right)$ can be approximatelyestimated via GA method.

\section{CONSTRUCTION OF POLAR CODES based on AWGN and Rayleigh fading channel:}

AWGN Channel The input-output relationship for an additive white Gaussian noise (AWGN) channel can be expressed by

$$
y=x+n
$$

Where $\left(3^{5}\right)$ is the transmitted source signal, $y$ denotes the output of the channel, $n$ is random noise variable with zero mean, variance

$$
\begin{aligned}
& \sigma^{2}=E\left[n^{2}\right] \\
& \quad p(y / x)=\frac{1}{\sqrt{2 \pi \sigma^{2}}} \exp \left(-\frac{n^{2}}{2 \sigma^{2}}\right)
\end{aligned}
$$

Since $n=y-x$, eqn. (7) can be re written as

$$
p(y / 4))=\frac{1}{\sqrt{2 \pi \sigma^{2}}} \exp \left(-\frac{(y-x)^{2}}{2 \sigma^{2}}\right)
$$

In case of BPSK modulation $x \in(+1,-1)$. Hence the LLR is computed by

$$
\begin{aligned}
L(y) & =\log \frac{p(y / x=+1)}{p(y / x=-1)} \\
& =\log \left(\frac{p(y / x)=\frac{1}{\sqrt{2 \pi \sigma^{2}}} \exp \left(-\frac{(y-1)^{2}}{2 \sigma^{2}}\right)}{p(y / x)=\frac{1}{\sqrt{2 \pi \sigma^{2}}} \exp \left(-\frac{(y+1)^{2}}{2 \sigma^{2}}\right)}\right) \\
= & \frac{2 y}{\sigma^{2}}
\end{aligned}
$$

\section{D.Rayleigh Fading Channel}

The input-output relationship for Rayleigh fading channel is given by

$$
y=h x+n
$$

Where $h$ is a random variable with Rayleigh PDF. $\mathrm{n}$ is the AWGN with zero mean and variance $=E\left[\sigma^{2}\right]_{5}$ ) For Rayleigh fading channel, the transition probability can be expressed by

$$
p(y / x)=\frac{1}{\sqrt{2 \pi \sigma^{2}}} \exp \left(-\frac{1}{2} \frac{n^{2}}{\sigma^{2}}\right)
$$

Since $n=y-h x \quad$ eqn. (6) can be written as 


$$
p(y / x)=\frac{1}{\sqrt{2 \pi \sigma^{2}}} \exp \left(-\frac{1}{2} \frac{(y-h x)^{2}}{\sigma^{2}}\right)
$$

For BPSK modulation $x \in(+1,-1)$. Assuming $\mathrm{h}$ is perfectly known at the receiver, the log likelihood ratio (LLR) is calculated as

$$
L(y)=\frac{2 h y}{\sigma^{2}}
$$

According to the presentation in (4), minimizing the exactBLER is equivalent to minimizing $P\left(C_{i}\right) \mathrm{s}$. Hence, to constructa polar code with rate $R=K / N$, where $K$ is the numberof information bits, we should select $K$ bit-channels with thelowest $P\left(C_{i}\right)$

s. Fortunately, because $P\left(C_{i}\right)$ s can be computed by(5) which is monotonically decreasing, we only need to selectthe bit-channels corresponding to the $K$ largest $E\left[L_{N}^{(i)}\right] \mathrm{s}$.

From now on, we compare the complexities of the threeconstruction methods. They are the Monte Carlo method in[1], the density evolution method in [3]-[5] and the GA-based

method proposed in this paper.

The successive cancellation decoding (SCD) algorithm essentially follows the same encoder diagram (butterfly diagram). The likelihood ratios (LRs) are propagated from right to left on the butterfly diagram. The node at upper corner of the butterfly is called the upper node and node at the lower corner of the butterfly is the lower node. The LRs at each node are calculated for decoding properly.

The likelihoods evolve in the reverse direction from rightto-left. The likelihood ratio is defined as

$$
\begin{aligned}
& L_{N}^{(i)}\left(y_{1}^{N}, u_{1}^{i-1}\right)=\frac{w_{N}^{i}\left(y_{1}^{N}, u_{1}^{i-1} \mid u_{i}=0\right)}{w_{N}^{i}\left(y_{1}^{N}, u_{1}^{i-1} \mid u_{i}=1\right)} \\
& u_{i}=\left\{\begin{array}{lll}
0 & \text { if } & L_{N}^{(i)}\left(y_{1}^{N}, u_{1}^{i-1}\right) \geq 1 \\
1 & \text { otherwise }
\end{array}\right.
\end{aligned}
$$

\section{RESULTS AND DISCUSSIONS}

In this section, the performance of polar codes over AWGN, Rayleigh fadingare evaluated through simulations using MATLAB for code lengths 128 , with the code rate $\mathrm{R}=0.5$ corresponding to message lengths 64,128 , and 256, respectively.The BER vsE $E_{b} / N_{o}$ performance of the polar codes over AWGN and Rayleigh fading channels are shown in Figures 1.

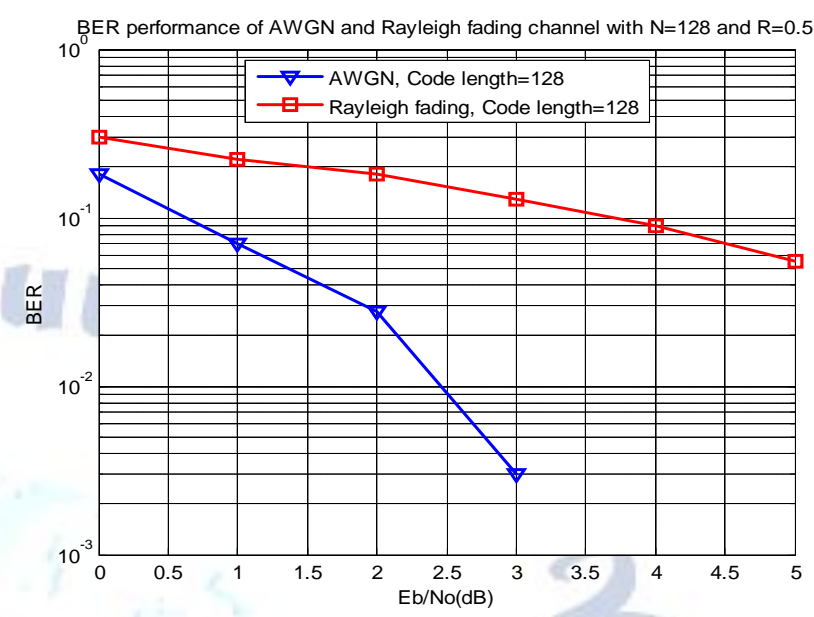

Figure 1 BER performance of AWGN and Rayleigh fading channel with $\mathrm{N}=128$ and $\mathrm{R}=\mathbf{0 . 5}$

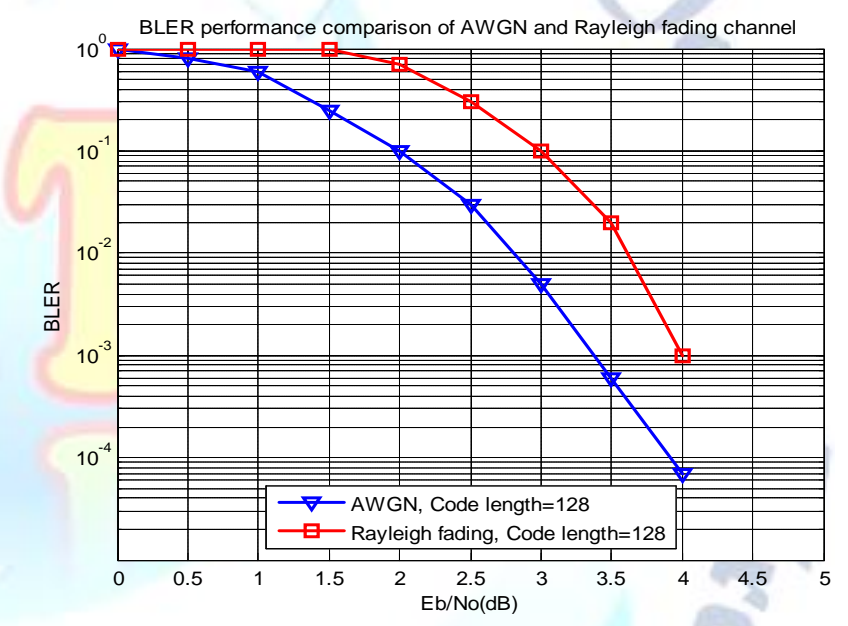

Figure 2: BLER performance comparison of polar codes constructed via AWGN and Rayleigh fading channels with $\mathrm{N}=128$ and $\mathrm{R}=0.5$.

The BER and BLER values are computed by averaging over 1000 independent trials for all the considered channels. From Figures $\mathbf{1}$, it is observed that BER performance over AWGN channel outperforms the performance of Rayleigh fading channels for short messages.

Figure 2 shows the BLER performance of polar codes constructed by GA and montecarlo method with respect to AWGN and Rayleigh fading channels with $\mathrm{N}=128$ and $\mathrm{R}=0.5$ respectively. In the simulation, SC decoder is employed. It illustrates that the performance of the polar codes constructed by GA has almost the same performance compared with those constructed by montecarlo method with short message transmissions. 


\section{CONCLUSIONS}

This paper has presented performance evaluation for polar codes over different channel models AWGN, Rayleigh fading channel for 5G short messages transmission. From simulation results, it is observed that the AWGN channel outperforms the Rayleigh fading channel for short message transmission. The same concept extended to different channel models with respected to GA and Monto Carlo methods for 5G short message transmissions. Then, a lower complexity construction method is described to minimize the BLER here. In other words, if we want to improve the performance of polar codes further, one possible choice is designing a more reliable decoding algorithm and then design a proper construction method suitable for that decoding algorithm. This is also our future work.

\section{REFERENCES}

[1] Murali K., Prasuna K. (2020) Survey on Saliency-Based Approach of Error Correction for 5G Communication. In: Satapathy S., Bhateja V., Mohanty J., Udgata S. (eds) Smart Intelligent Computing and Applications. Smart Innovation, Systems and Technologies, vol 160. Springer, Singapore

[2] RMori.etc.al,"Performance of polarcodes with theconstruction using densityevolution,"

IEEECommunicationsLetters, vol3, no7,pp519-521,Jul2009.

[3] ITal.etc.al, "How to Construct Polar Codes," InformationTheory, IEEETran's ,vol59, no10, pp6562-6582, Oct2013.

[4] [PTrifonov.etc.al, "Efficient Design and Decoding of Polar Codes," IEEE Tran's on Communications, vol60, no11, pp3221-3227, November2012.

[5] DWu.etc.al, "Construction and Block Error Rate Analysis of Polar Codes OverAWGN Channel Based on Gaussian Approximation," IEEE Commun's-Letters, vol18, no7, pp1099-1102, Jul2014.

[6] KChen.etc.al,"ImprovedSuccessiveCancellation Decoding ofPolarCodes,"IEEE-Tran's onCommun's, vol61, no8, pp3100-3107, August2013.

[7] YLi, "Pilot-symbol-aided channel estimation for ofdm in wireless systems," IEEE Tran's onVehicularTechnology, vol49, no4, pp12071215, Jul2000.

[8] MMorelli.etc.al, "AComparisonofPilotAidedChannelEstimat ion Methods for OFDMSystems," IEEETransa'sSignalProcessing, vol. 49, no12, pp3065-3073, Dec2001.

[9] YLi, "Simplified channel estimation for ofdm systems with multiple transmit antennas," IEEE-Transa's on Wireless-Commun's, vol1,no1,pp67-75,Jan2002. 